\title{
Receiver positioning using received signal strength
}

\author{
Chuanxue Jin, Xiaoning Huanga), Zhi Chen, and Wanbin Tang \\ National Communications Lab., University of Electronic Science and Technology \\ of China, Chengdu 611731, China
}

a)hxn_1989@163.com

\begin{abstract}
In wireless positioning, estimating the location of a receiver is extremely challenging since the receiver does not transmit signal and it is invisible to sensors. To deal with the issue, we proposes a received signal strength (RSS)-based method to position the receiver based on the signal from the corresponding transmitter. Our results indicate that the proposed method has about 20 40 meters positioning error in average, which reaches the same level of the positioning error as the conventional RSS-based Tx positioning methods.
\end{abstract}

Keywords: estimation, receiver, RSS, wireless positioning Classification: Sensing

\section{References}

[1] G. Mao and B. Fidan, Localization algorithms and strategies for wireless sensor networks, 2009.

[2] X. Li, "RSS-based location estimation with unknown path loss model," IEEE Trans. Wireless Commun., vol. 5, no. 12, pp. 3626-3633, Dec. 2006. DOI: 10.1109/TWC.2006.256985

[3] R. M. Vaghefi, M. R. Gholami, R. M. Buehrer, and E. G. Strom, "Cooperative received signal strength-based sensor localization with unknown transmit powers," IEEE Trans. Signal Process., vol. 61, no. 6, pp. 1389-1403, Mar. 2013. DOI:10.1109/TSP.2012.2232664

[4] R. Zhang, "On active learning and supervised transmission of spectrum sharing based cognitive radios by exploiting hidden primary radio feedback," IEEE Trans. Commun., vol. 58, no. 10, pp. 2960-2970, Oct. 2010. DOI:10.1109/ TCOMM.2010.082710.090412

[5] E. Azzouz and A. Nandi, Automatic modulation recognition of communication signals, 2010.

[6] B. Ramkumar, "Automatic modulation classification for cognitive radios using cyclic feature detection," IEEE Circuits Syst. Mag., vol. 9, no. 2, pp. 27-45, Jun. 2009. DOI:10.1109/MCAS.2008.931739

[7] A. H. Sayed, A. Tarighat, and N. Khajehnouri, "Network-based wireless positioning: challenges faced in developing techniques for accurate wireless location information," IEEE Signal Process. Mag., vol. 22, no. 4, pp. 24-40, Jul. 2005. DOI:10.1109/MSP.2005.1458275

[8] 3GPP TR 25.814, "Physical layer aspects for evolved universal terrestrial radio access (UTRA)," 2006. 


\section{Introduction}

Positioning an unknown node has been extensively studied in the past decades [1], which is the essential technique for location-aware applications. Fig. 1(a) provides a conventional wireless positioning scenario: some fixed sensors with known locations (called anchors) measure the transmitted signal from an unknown node (called target), then they estimate the location of the target. In existing literatures, positioning algorithms include received signal strength (RSS) [2,3], time of arrival (TOA), time difference of arrival (TDOA), angle of arrival (AOA), etc. However, they all belong to transmitter positioning technique since they treat the unknown transmitter as the target.

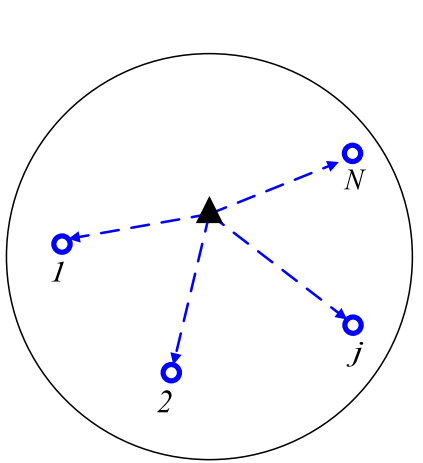

A Tx (Anchor)

- Sensor

(a) The conventional transmitter positioning
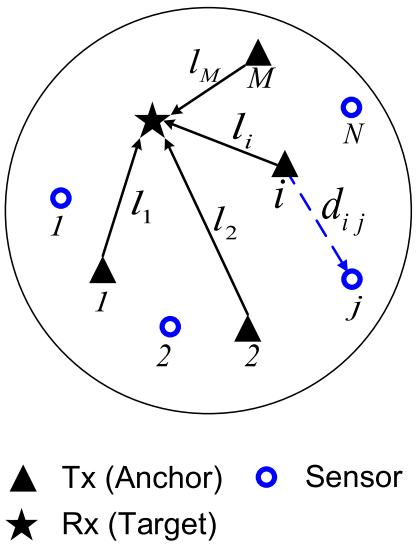

$\Delta$ Tx (Anchor) 0 Sensor $\star \mathrm{Rx}($ Target)

(b) The proposed receiver positioning

Fig. 1. System model.

In contrast, few contributions discuss receiver positioning technique since the receiver works in a passive way and it is invisible to sensors, i.e., the receiver does not transmit signal during the reception ${ }^{1}$. Therefore, receiver positioning becomes extremely challenging. This significantly impedes the development of the locationaware applications.

In this paper, we proposes an RSS-based receiver positioning method under the scenario $^{2}$ that multiple transmitters communicate with a common receiver using time division multiple access (TDMA) protocol. In the proposed method, we treat the multiple transmitters as the anchors, and position the receiver by estimating the transmitter-to-receiver (Tx-Rx) distances, i.e., Anchor-Rx distances. It is reasonable to treat those transmitters as the anchors as the location of the transmitters can be obtained using the conventional transmitter positioning methods [1]. Regarding the Tx-Rx distance estimation, our method exploits the close-loop power control

\footnotetext{
${ }^{1}$ Even though the receiver may feedback information to its transmitter, it goes through another frequency band in frequency division duplex (FDD) systems. Thus, the receiver is still invisible (or silent) to the sensors since the feedback channel may not be known to the sensors.

${ }^{2}$ Even though this letter considers the many-to-one scenario, the proposed method can also be used in the point-topoint scenario when the transmitter is moving. This is because during the geographic travel of the transmitter, the sensors can obtain multiple locations of the transmitter as well as the corresponding distances to the receiver.
} 
(CLPC) between the transmitters and the receiver, which has been widely used in modern wireless communication systems. Under CLPC, the power adjustment of the transmitters actually carries the Tx-Rx distance information. Therefore, by measuring the transmission power of the transmitters, the sensors are able to estimate the Tx-Rx distances and then obtain the location of the corresponding receiver.

\section{System model}

Fig. 1(b) provides the system model of this paper, where $M$ transmitters (labeled as Tx), a receiver (labeled as $\mathrm{Rx}$ ), and $N$ sensors are uniformly distributed on a disk with the radius $R$. Here, we denote $l_{i}$ as the distance between the $i$-th Tx (denoted as $\left.\mathrm{Tx}_{i}\right)$ and the receiver, and $d_{i j}$ as the distance between the $i$-th Tx and the $j$-th sensor (denoted as Sensor ${ }_{j}$ ). In particular, we assume that the $\mathrm{Tx}_{i}$ 's location and the corresponding $\mathrm{Tx}_{i}$-Sensor ${ }_{j}$ distances $d_{i j}$ are known to the sensors. Next, we provide the signal models for the Tx-Rx and Tx-Sensor links, respectively.

- Tx-Rx Links: We denote $g_{i}$ and $q_{i}(k)$ as the path-loss and shadowing coefficients between the $\mathrm{Tx}_{i}$ and the $\mathrm{Rx}$, where the former is modeled as $g_{i}=C / l_{i}^{\alpha}$ ( $C$ is a constant and $\alpha$ is the path-loss coefficient) and the latter follows log-normal distribution with the standard deviation of $\delta$. Here, $k(1 \leq k \leq K)$ is the index of independent shadowing. In static scenarios, we have $K=1$ during the positioning. In time-varying scenarios, the shadowing coefficient varies with time, and then the $\mathrm{Tx}_{i}$ and the Rx experiences $K>1$ independent shadowing coefficients during the positioning.

Let $p_{i}$ as the transmission power of the $\mathrm{Tx}_{i}$, then the average SNR of the $\mathrm{Rx}$ served by the $\mathrm{Tx}_{i}$ can be expressed as $\gamma_{i}(k)=p_{i} g_{i} q_{i}(k)$, where the variance of the noise at the $\mathrm{Rx}$ is normalized. When we consider the system with CLPC ${ }^{3}$, the $\mathrm{Tx}_{i}$ automatically adjusts the transmission power to meet a certain target SNR at the Rx, denoted as $\Gamma^{4}$. Then, the transmission power of $\mathrm{Tx}_{i}$ can be obtained by

$$
p_{i}=\frac{\Gamma}{g_{i} q_{i}(k)} .
$$

- Tx-Sensor Links: Similarly, we denote $g_{i j}^{\prime}$ and $q_{i j}^{\prime}(k)$ as the path-loss and shadowing coefficients between the $\mathrm{Tx}_{i}$ and the Sensor ${ }_{j}$, which follow the same model as in Tx-Rx links, then the average SNR at the Sensor $_{j}$ can be expressed as

$$
\gamma_{i j}^{\prime}(k)=p_{i} g_{i j}^{\prime} q_{i j}^{\prime}(k),
$$

where the variance of the noise at the Sensor ${ }_{j}$ is normalized. When adopting the path-loss model $g_{i j}^{\prime}=C / d_{i j}^{\alpha}$ and substituting (1) into (2), we can rewrite (2) as

$$
\gamma_{i j}^{\prime}(k)=\Gamma \frac{q_{i j}^{\prime}(k)}{q_{i}(k)}\left(\frac{l_{i}}{d_{i j}}\right)^{\alpha} .
$$

\footnotetext{
${ }^{3}$ Since this paper focuses on proposing the basic idea of Rx positioning, we consider the perfect CLPC [4], where the quantization error and time delay are not considered.

${ }^{4}$ Even though $\Gamma$ is the target SNR of the Rx, it can still be obtained by the Sensor ${ }_{j}$ using blind signal processing technique, e.g., recognizing the modulation level of the $\mathrm{Tx}_{i}$ 's signal and obtaining the corresponding target SNR $[5,6]$.
} 


\section{RSS-based receiver positioning}

\subsection{Estimate the Tx-Rx distance}

In this subsection, we first design a ML estimator to let each sensor obtain the $\mathrm{Tx}_{i^{-}}$ $\mathrm{Rx}$ distance. To facilitate the derivation, we rewrite (3) using $\mathrm{dB}$ unit and then obtain the average SNR at the Sensor $_{j}$ as

$$
\gamma_{i j[\mathrm{~dB}]}^{\prime}(k)=10 \alpha \log _{10}\left(\frac{l_{i}}{d_{i j}}\right)+q_{i j[\mathrm{~dB}]}^{\prime}(k)-q_{i[\mathrm{~dB}]}(k)+\Gamma_{[\mathrm{dB}]} .
$$

Here, $l_{i}$ is the unknown Tx-Rx distance to be estimated. $q_{i j[\mathrm{~dB}]}^{\prime}(k)$ and $q_{i[\mathrm{~dB}]}(k)$ are independent shadowing coefficients, which follow normal distribution in $\mathrm{dB}$ unit, i.e., $q_{i j[\mathrm{~dB}]}^{\prime}(k) \sim N\left(0, \delta^{2}\right)$ and $q_{i[\mathrm{~dB}]}(k) \sim N\left(0, \delta^{2}\right)$. Then, we have $q_{i j[\mathrm{~dB}]}^{\prime}(k)-$ $q_{i[\mathrm{~dB}]}(k) \sim N\left(0,2 \delta^{2}\right)$.

If the sensor can obtain $K$ average SNR with independent shadowing coefficients, then we can obtain the K-dimension conditional probability density function (PDF) as

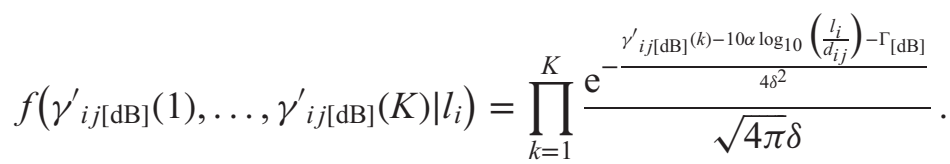

Using the standard maximum likelihood distance estimator, then the Sensor ${ }_{j}$ can obtain the $\mathrm{Tx}_{i}-\mathrm{Rx}$ distance as

$$
\hat{l}_{i}=d_{i j} \cdot 10^{\frac{1}{10 K \alpha} \sum_{k=1}^{K}\left(\gamma_{i j \mathrm{dBB}]}^{\prime}(k)-\Gamma_{[\mathrm{dB}]}\right)} .
$$

Like other RSS-based distance estimators, the estimation in (6) is biased, i.e.,

$$
\mathbf{E}\left\{\hat{l}_{i}\right\}=\mathbf{E}\left\{d_{i j} \cdot 10^{\frac{1}{10 K \alpha} \sum_{k=1}^{K}\left(\gamma_{i j[\mathrm{~dB}]}^{\prime}(k)-\Gamma_{[\mathrm{dBB}]}\right)}\right\}=l_{i} \cdot e^{\frac{\delta^{2}}{K \xi^{2}}}
$$

where $\xi=10 K \alpha / \ln 10$. By modifying the estimator, we can obtain the unbiased estimation as follows

$$
\hat{l}_{i}=d_{i j} \cdot e^{-\frac{\delta^{2}}{K \xi^{2}}} \cdot 10^{\frac{1}{10 K \alpha} \sum_{k=1}^{K}\left(\gamma_{i j \mathrm{~dB}]}^{\prime}(k)-\Gamma_{[\mathrm{dB}]}\right)} .
$$

\subsection{Positioning the receiver}

Once we obtain the Tx-Rx distance, positioning the receiver becomes easy. Here, we adopt the conventional linear method to estimate the Rx location [7]. Specifically, we first derive the positioning method for one sensor, denoted as Sensor ${ }_{j}$, and then obtain the Rx location using $N$ sensors.

Let $\left(x_{0}, y_{0}\right)$ and $\left(x_{i}, y_{i}\right)$ as the locations of the $\mathrm{Rx}$ and $\mathrm{Tx}_{i}$, respectively, where $i=1,2, \ldots, M$. We further let $D_{i}=x_{i}^{2}+y_{i}^{2}$, then we have

$$
\mathbf{H X}=\mathbf{b} \text {, }
$$

where

$$
\mathbf{H}=\left[\begin{array}{cc}
\left(x_{2}-x_{1}\right) & \left(y_{2}-y_{1}\right) \\
\vdots & \vdots \\
\left(x_{M}-x_{1}\right) & \left(y_{M}-y_{1}\right)
\end{array}\right], \mathbf{X}=\left[\begin{array}{c}
x_{0} \\
y_{0}
\end{array}\right], \mathbf{b}=\mathbf{0 . 5}\left[\begin{array}{c}
D_{2}-D_{1}-\left(l_{2}^{2}-l_{1}^{2}\right) \\
\vdots \\
D_{M}-D_{1}-\left(l_{M}^{2}-l_{1}^{2}\right)
\end{array}\right] .
$$


Once solving the equation (9), we can obtain the location of the $\mathrm{Rx}$ as

$$
\hat{\mathbf{X}}=\left[\begin{array}{c}
\hat{x}_{0} \\
\hat{y}_{0}
\end{array}\right]=\left(\mathbf{H}^{\mathrm{T}} \mathbf{H}\right)^{-1} \mathbf{H}^{\mathrm{T}} \mathbf{b} .
$$

When we consider $N$ sensors that can share their distance estimations, they can obtain the average Tx-Rx distance by

$$
\bar{l}_{i}=\frac{1}{N} \sum_{j}^{N} \hat{l}_{i}^{(j)} .
$$

Substituting $\bar{l}_{i}$ into (10), we can obtain the Rx's location more accurately.

\section{Simulation results}

In this section, we demonstrate the performance of the proposed RSS-based receiver positioning method. Here, $M$ Txs, a Rx, and $N$ sensors are uniformly distributed on the disk with the radius $R=100 \mathrm{~m}$. In the simulation, the system bandwidth is $B=10 \mathrm{MHz}$, the power spectrum density of the noise is $N_{0}=$ $-174 \mathrm{dBm}$, and the target SNR of the Rx is $\Gamma=10 \mathrm{~dB}$. In wireless channels, $C=-128.1 \mathrm{~dB}$ and $\alpha=3.76$ are adopted according to [8]. Furthermore, $10^{4}$ Monte Carlo trails are conducted for each simulation curve.

To evaluate the positioning performance, we define the root-mean-square error $(\mathrm{RMSE})$ as $\mathrm{RMSE}=\sqrt{\left(\hat{x}_{0}-x_{0}\right)^{2}+\left(\hat{y}_{0}-y_{0}\right)^{2}}$.

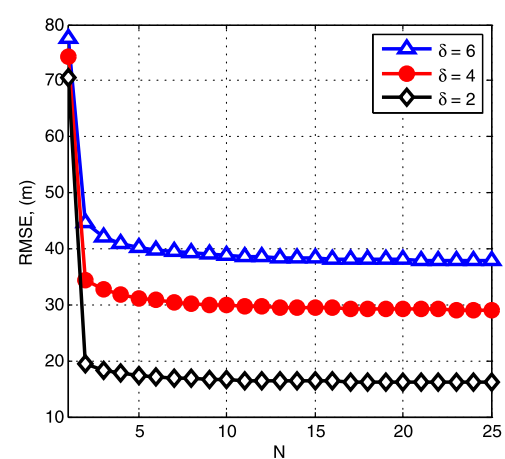

(a) RMSE versus $\mathrm{N}$

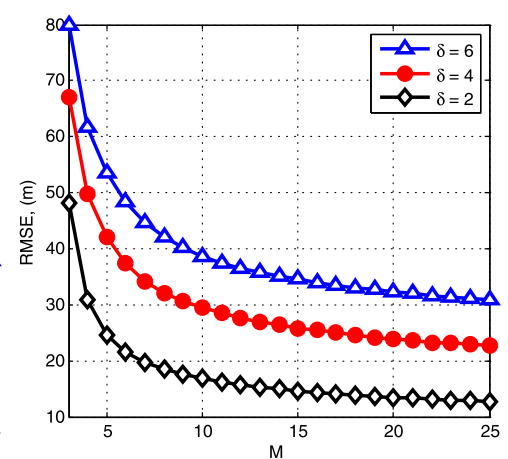

(b) RMSE versus $M$

Fig. 2. Impact of $N$ and $M$ on the RMSE of Rx positioning.

In Fig. 2, we consider the static scenario, where $K=1$. We assume that the number of Txs $M=10$ in Fig. 2(a) and the number of sensors $N=10$ in Fig. 2(b). From the two figure, we find that the number of Txs $(M)$ dominates the RMSE as the number of sensors $(N)$ is larger than 5, i.e., $N \geq 5$. This is because we treat the $\mathrm{Tx}$ as anchor and more anchors result in a better performance. In addition, we compare the curves with different standard deviations of the shadowing. The RMSE reduces as the standard deviation $\delta$ decreases from 6 to 2. This is because the small $\delta$ introduces less uncertainty into the wireless channel.

Fig. 3 illustrates the RMSE of the proposed method versus the number of independent shadowing coefficients $(K)$, where $M=10$ Txs and $N=10$ sensors are considered. Here, when $K>1$, it is the time-varying scenario, which means that 


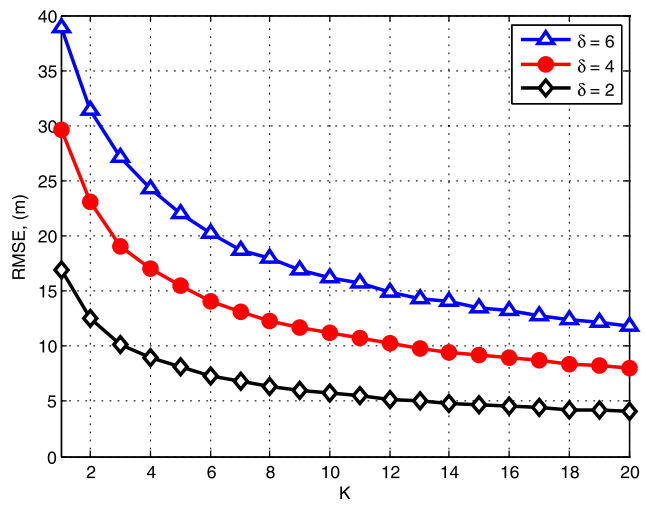

Fig. 3. Impact of $K$ on the RMSE of Rx positioning.

each node experiences $K$ independent shadowing coefficients during the Rx positioning. From the figure, the RMSE reduces as $K$ increases from 1 to 20 . The reason is that the uncertainty of the shadowing can be reduced by considering multiple independent shadowing coefficients. In particular, the proposed method has about $20 \sim 40 \mathrm{~m}$ positioning error, where the Rx is uniformly located on a disk with the radius $100 \mathrm{~m}$. This means that the proposed Rx positioning method actually reaches the same level of the positioning error as the conventional RSSbased Tx positioning methods. For example, the method in [2] has about $6 \mathrm{~m}$ positing error, where the Tx is uniformly located on a disk with the radius $20 \mathrm{~m}$.

\section{Conclusions}

In this paper, we proposed an RSS-based receiver positioning method to estimate the location of an unknown receiver. Different from existing RSS-based positioning, our method treated the associated transmitters as the anchors, and conducted the positioning by estimating the distances between the anchors and the receiver. The simulation results showed the effectiveness of the proposed method.

With our method, the location-aware applications can be extended to many scenarios. For example, a scenario where multiple wireless networks may share the same frequency band, the proposed method enables one system to locate the other base stations. This provides the crucial information for co-channel interference management.

\section{Acknowledgments}

This paper is supported in part by the grant from Science and Technology on Information Transmission and Dissemination in Communication Networks Laboratory and by the National Natural Science Foundation of China (NS-FC) under Grants 61271169 . 